\title{
Automatic activation of categorical and abstract analogical relations in analogical reasoning
}

\author{
ADAM E. GREEN, JONATHAN A. FUGELSANG, and KEVIN N. DUNBAR \\ Dartmouth College, Hanover, New Hampshire
}

\begin{abstract}
We examined activation of concepts during analogical reasoning. Subjects made either analogical judgments or categorical judgments about four-word sets. After each four-word set, they named the ink color of a single word in a modified Stroop task. Words that referred to category relations were primed (as indicated by longer response times on Stroop color naming) subsequent to analogical judgments and categorical judgments. This finding suggests that activation of category concepts plays a fundamental role in analogical thinking. When colored words referred to analogical relations, priming occurred subsequent to analogical judgments, but not to categorical judgments, even though identical four-word stimuli were used for both types of judgments. This finding lends empirical support to the hypothesis that, when people comprehend the analogy between two items, they activate an abstract analogical relation that is distinct from the specific content items that compose the analogy.
\end{abstract}

Analogical reasoning has been regarded as a key component of intelligence (Sternberg, 1977), inductive reasoning (Holyoak \& Thagard, 1997), and everyday discourse (Blanchette \& Dunbar, 2002), as well as learning, understanding our environment, and generating novel ideas (Dunbar \& Blanchette, 2001; Holyoak, 2005). In order to understand or generate an analogy, such as "a hand is to a glove as a foot is to a sock," a person must form a connection, called a mapping, between the abstract structure of one item or situation and the abstract structure of another item or situation. Mapping one structure onto another is based on forming one-to-one alignments between elements of the two structures (Gick \& Holyoak, 1980; Markman \& Gentner, 2000) as, in the example above, hand is aligned with foot and glove is aligned with sock. In the present investigation, we sought to identify the role of categorization (e.g., hand and foot are body parts; glove and sock are articles of clothing) as a mechanism for aligning terms in analogical mapping. Additionally, we sought to determine whether the mental representation of an analogical relation can be distinguished from the component terms that make up the analogy.

\section{Categorization in Analogical Reasoning}

Despite the importance of analogical thinking, several key questions remain regarding the mappings that tie analogous items together in the mind. One key question concerns the role of categorization in analogical mapping.

This research was funded by a grant to K.N.D. from Dartmouth College. The authors thank D. Kraemer for thoughtful comments on earlier versions of this manuscript. Correspondence should be addressed to A. E. Green, Department of Psychological and Brain Sciences, Dartmouth College, 6207 Moore Hall, Hanover, NH 03755 (e-mail: adam .e.green@dartmouth.edu).
Many researchers have suggested that categorization may be importantly related to analogical reasoning (Bowdle \& Gentner, 2005; Gentner \& Markman, 1997; Hesse, 1966; Holyoak \& Thagard, 1997; Sternberg, 1977). Generally, analogy research has treated categorization as an end result of analogical reasoning. Gentner and Markman (1997), for example, argue that determining that two items or situations are analogous is an important criterion in deciding that the two entities are members of a common category.

A distinct, nonconflicting hypothesis is that categorization serves to align structural elements one-to-one so that analogical mapping can occur (Green, Fugelsang, Kraemer, Shamosh, \& Dunbar, 2006). Similar notions have been suggested by other researchers. Gick and Holyoak (1983) noted that "mapped elements ... are typically similar but not identical" (p. 6). Bassok and colleagues (Bassok, Chase, \& Martin, 1998; Wisniewski \& Bassok, 1999) demonstrated that categorically related items, such as apples and oranges, can be readily compared because category comembership makes them alignable with each other. However, empirical investigation has not yet been directed toward categorization as a possible mechanism for aligning component terms during analogical mapping.

To investigate this issue, we employed four-word analogies, a mainstay of academic and intelligence tests (Sternberg, 1977). Four-word analogies, such as the one composed by the word pairs hand:glove +foot:sock, have a consistent and readily decomposable structure. Between the two terms of each word pair is a semantic connection, called a conventionalized semantic relation, which describes what the two items have to do with each other (e.g., hand wears glove; foot wears sock). In order to comprehend the analogy (hand is to glove as foot is to sock), an analogical mapping must be formed between the two word pairs. 
A key point to consider with regard to four-word analogies is that not all four-word sets that contain two conventionalized semantic relations constitute analogies. Fourword sets, such as copier:document + rowboat:pier, have conventionalized semantic relations within each word pair but do not constitute an analogy because the elements of the two word pairs do not align with each other. Green et al. (2006) have suggested that the process of aligning elements of a four-word analogy is facilitated by finding categorical relations between elements. For example, in the four-word set hand:glove +foot:sock, hand and foot are comembers of a category (parts of the body). Glove and sock are also comembers of a category (articles of clothing). Thus, the elements of these two word pairs can be aligned based on category. As noted above, forming oneto-one alignments is fundamental to the mapping process by which analogies are constructed.

The connection between categories and analogies has been noted by many researchers (Bowdle \& Gentner, 2005; Gentner \& Markman, 1997; Hesse, 1966; Holyoak \& Thagard, 1997; Sternberg, 1977); however, no specific role has yet been empirically determined for categorization in analogical mapping. In the present investigation, we sought to test the hypothesis that the one-to-one alignment of terms necessary for analogical mapping involves grouping the terms on the basis of category comembership.

\section{Priming of Analogical Relations}

Priming of analogously related items has been shown in a number of studies using a variety of research methodologies. The typical finding is that processing analogical relations between two situations or word pairs facilitates performance in cognitive tasks. Spellman, Holyoak, and Morrison (2001) demonstrated facilitated processing of single word pairs that had been analogically primed by preceding word pairs. For example, these authors found that the word pair bear:cave primed the analogically related word pair bird:nest, more than the analogically unrelated word pair bear:swamp. Blanchette and Dunbar (2002) identified priming of analogically related concepts in text processing. Several other investigations have also demonstrated that similarity of internal relations enables one item or situation to facilitate memory access to a second item or situation (Gentner, Rattermann, \& Forbus, 1993; Holyoak \& Koh, 1987; Keane, 1988; Ross, 1989; Schunn \& Dunbar, 1996; Wharton, Holyoak, \& Lange, 1996).

These investigations have demonstrated priming of analogical relations when individual terms that compose an analogy prime other, analogously related terms. However, a key question remains as to whether the abstract analogical relation is mentally represented only by component terms that make up the analogy. Alternatively, the abstract analogical relation itself may have a mental representation that is distinguishable from the component terms. In the present investigation, we sought to address this question by determining whether the abstract analogical relation itself was subject to priming independently of the component terms.

\section{The Approach of the Present Investigation}

Color-naming paradigms modified from the classic Stroop task constitute a family of experimental paradigms that is sensitive to priming effects in general (Catena, Fuentes, \& Tudela, 2002; Marí-Beffa, Estévez, \& Danziger, 2000) and to conceptual priming in particular (Pritchard \& Neumann, 2004; Segal, Gemar, Truchon, Guirguis, \& Horowitz, 1995). In these paradigms, activating the mental representation of a concept makes subjects more predisposed to read a word that is semantically related to the concept. This predisposition to read the word interferes with naming the ink color of the word. In these paradigms, stronger priming effects are indicated by slower response times (RTs) on the color-naming task. In the present investigation, we used a Stroop color-naming paradigm to assess the extent to which processing fourword sets results in activation of categorical and abstract analogical concepts. The subjects named the color of a single word immediately after each four-word set.

In order to examine the activation of category concepts in analogical reasoning, we compared the standard fourword analogy task with a second four-word task. This task did not require comprehension of the four words as a single analogy. Rather, it required explicit identification of category relations between individual words. We used identical four-word stimuli in the analogy and category tasks in order to make the comparison as clear as possible. If analogical mapping requires categorization, then category concepts should be activated not only by the category task but should also be activated by the analogy task.

The use of identical stimuli in the analogy and category tasks also helped us to distinguish the mental representation of abstract analogical relations from the component terms that make up the analogies. If the component terms are sufficient to represent the abstract analogical relation, then priming of the analogical relation should occur equally in the categorical and analogical tasks. However, if the analogy task primes the abstract analogical relation, but the category task (using the same four-word set) does not, this would indicate that the component terms alone are not sufficient to represent the abstract analogical relation. Also, if the mental representation of an abstract analogical relation is distinguishable from the component terms, it should be possible to measure the priming of the abstract analogical relation by using a colored word that refers directly to the relation itself (not to any of the component terms).

\section{METHOD}

\section{Subjects}

Thirty-six undergraduate right-handed native English speakers (20 females, 16 males; age range, $18-24$ years) took part in the investigation for course credit.

\section{Materials}

Stimuli in each trial consisted of a four-word set followed by a single colored word. Figure 1 provides examples of stimuli used in the different types of trials, and a full list of stimuli is provided in the Appendix. Stimuli were designed by the experimenter in order to en- 


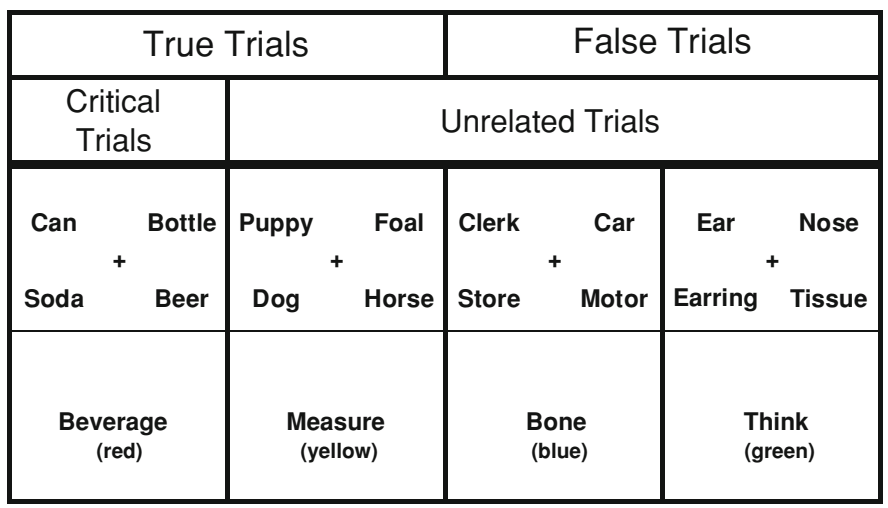

Figure 1. Example stimuli. The parenthetical color names below the words in the bottom row of the figure represent the four colors in which colored words were presented. These parenthetical color names did not appear in the actual stimuli.

sure that the words that composed the four-word sets were related to each other in specific ways. On all true trials, the relations between the words of the four-word set represented two conventionalized semantic relations as well as two category relations and an analogy relation (see Figure 2 for a schematic representation of the relations contained in four-word sets on true trials). All four-word sets were previously determined to be true or false at a level of $\geq 90 \%$ agreement among 27 Dartmouth College undergraduates. On false trials, the four-word set contained either one category relation $(50 \%)$ or no category relations $(50 \%)$, but never two category relations. False trials contained two conventionalized semantic relations ( $50 \%$ of false trials), one but not two conventionalized semantic relations $(25 \%)$, or no conventionalized semantic relations $(25 \%)$. The four-word set in false trials never represented an analogy relation.

The colored word that followed the four-word set was presented in red, yellow, blue, or green ( $25 \%$ in each color). In a subset of true trials called critical trials, the colored word referred either to one of the category relations in the preceding four-word set or to the analogy relation in the preceding four-word set $(50 \%$ of true trials were critical trials). On the other $50 \%$ of true trials, the colored word did not refer to any relation in the preceding four-word set. On false trials, the colored word did not refer to any relation present in the preceding four-word set. Trials in which the colored word did not refer to any relation in the preceding four-word set $(50 \%$ of true trials and all false trials) were called unrelated trials.

\section{Procedure}

Trials for both instruction types began with the presentation of a set of four words. The subjects were instructed to judge whether the four-word set included two conventionalized semantic relations (one in the left word pair and one in the right word pair). A conventionalized semantic relation was defined to exist "when there is a common sense way in which two things often do, or easily could, have to do with each other." If they judged that both conventionalized semantic relations were present, the subjects were instructed to make an additional judgment about that four-word set.

In the analogical (ANA) instruction type, the additional judgment was whether the four-word set constituted an analogy (between the left word pair and the right word pair). For example, given the fourword set gun:bullet+bow:arrow, the subjects judged that, since a gun shoots a bullet and a bow shoots an arrow, the two word pairs represent the same abstract relation and are thus analogous. In the categorical (CAT) instruction type, the additional judgment was whether two categorical relations were present within the four-word set (one in the top word pair and one in the bottom word pair). For example, given the four-word set gun:bullet+bow:arrow, the sub-
Category Instruction Type (CAT)

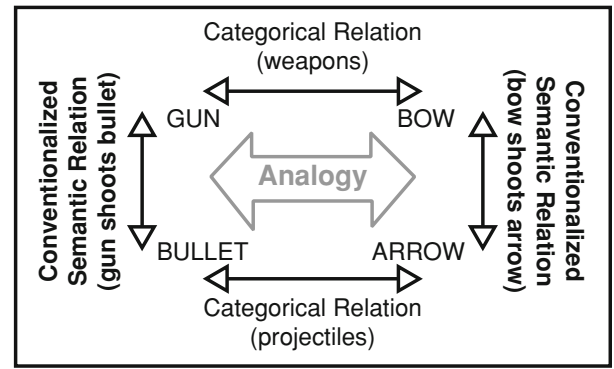

\section{Analogy Instruction Type (ANA)}

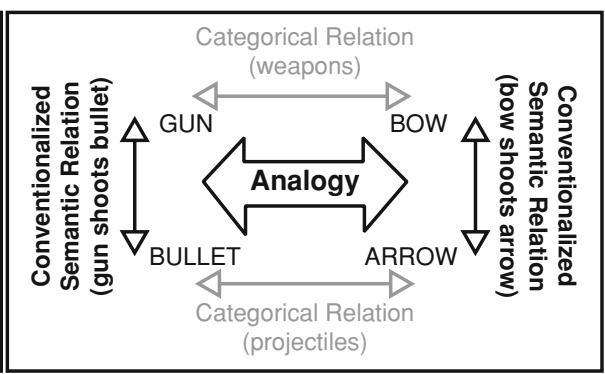

Figure 2. Relations involved in four-word stimuli in both instruction types. Stimuli in the CAT instruction type involved two conventionalized semantic relations and two categorical relations and constituted an overall analogical relation. However, the subjects were not explicitly instructed to evaluate the analogical relation constituted by the four terms. Stimuli in the ANA instruction type involved two conventionalized semantic relations and two categorical relations and constituted an overall analogical relation (gun is to bullet as bow is to arrow). However, the subjects were not explicitly instructed to evaluate the categorical relations. 
jects judged that both a gun and a bow are weapons and that both a bullet and an arrow are projectiles. Since stimuli were identical in the ANA and CAT instruction types, the instruction given to the subjects was the key difference.

In all trials, the four-word set was presented until the subjects responded "true" or "false" by buttonpress. The subjects were instructed that a four-word set was true if and only if all the relations delineated in the instructions were present in the four-word set; otherwise the four-word set was false.

Immediately upon the subjects' true/false response, the four-word set disappeared and a single colored word appeared at the center of the screen. This word remained on the screen until the subjects initiated a verbal color-naming response. Thus, each trial involved two separate stages: evaluation of a four-word set and Stroop colornaming of a presented word. RT for the Stroop color-naming task was the dependent variable in this experiment.

\section{Design and Apparatus}

A $2 \times 3$ mixed design was employed. The between-subjects variable was instruction type (CAT vs. ANA). The within-subjects variable was reference type (same, other, unrelated) of the colored word. As explained below, reference type depended on whether the colored word referred to the relation that the subjects had been instructed to identify (categorical or analogical) or to no relation in the four-word set. A total of 64 trials were presented to each subject. Half of these (32 trials) were true trials. That is, the four-word sets in these trials met the criteria for responding "true" according to the instructions (CAT or ANA instruction type, refer to Figure 2). Half of the true trials (16 trials) were critical trials. On critical trials, the colored word referred either to an analogy relation ( 8 trials) or a category relation ( 8 trials) that was present in the preceding four-word set.

Critical trials were identical in the two instruction types; that is, they were composed of the same four-word set followed by the same word in the same color. Critical trials were labeled by the instruction type in which they occurred and the reference type of the colored word. For example, when it occurred in the ANA instruction type, the four-word set painter:painting + sculptor:sculpture followed by the word create was labeled an ANA-same trial. The abstract concept of creating is what makes these two word pairs analogously similar, thus the colored word referred to the same relation (analogical) that the subjects were instructed to identify. In the CAT instruction type, this trial was labeled a CAT-other trial because the colored word did not refer to the relation that the subjects had been instructed to identify (categorical) but referred instead to the other relation (analogical). When it occurred in the ANA instruction type, painter:painting + sculptor:sculpture followed by the colored word artist was labeled an ANA-other trial. In the CAT instruction type, the same trial was labeled a CAT-same trial. All critical trials in both instruction types were either same trials or other trials. On unrelated trials, the colored word did not refer to a relation in the four-word set. Our analyses indicated that critical trial types were internally consistent. That is, RTs for each critical trial, averaged across subjects, were within 2.5 standard deviations of the mean RT for that trial type (CAT-same, CAT-other, ANA-same, ANA-other). All stimuli were presented on a G3 iMac computer running PsyScope 2.5.1 software (Cohen, MacWhinney, Flatt, \& Provost, 1993).

\section{RESULTS}

RTs greater than $2,000 \mathrm{msec}$ or less than $100 \mathrm{msec}$ were considered outliers and removed prior to analyses, resulting in elimination of $2.4 \%$ of responses. Figure 3 presents the mean RTs for critical trials. A $2 \times 3$ mixed ANOVA (instruction type [ANA, CAT] $\times$ reference type [same, other, unrelated]) revealed a main effect of reference type $\left[F(2,33)=19.00, M S_{\mathrm{e}}=6,059.28, p<.001\right.$, $\left.\eta^{2}=.35\right]$, no main effect of instruction type $[F(1,33)=$ $\left.2.32, M S_{\mathrm{e}}=35,843.85, p=.137, \eta^{2}=.064\right]$, and an interaction between instruction type and reference type $\left[F(2,33)=3.17, M S_{\mathrm{e}}=6,059.28, p<.05\right]$.

In order to further examine sources of the observed variance and assess the validity of our a priori predictions, independent $t$ tests were performed. A paired $t$ test revealed that ANA-other trials $(M=816 \mathrm{msec})$ were slower than unrelated trials $(M=741 \mathrm{msec})$ in the ANA

\section{Mean Color-Naming Response Times}

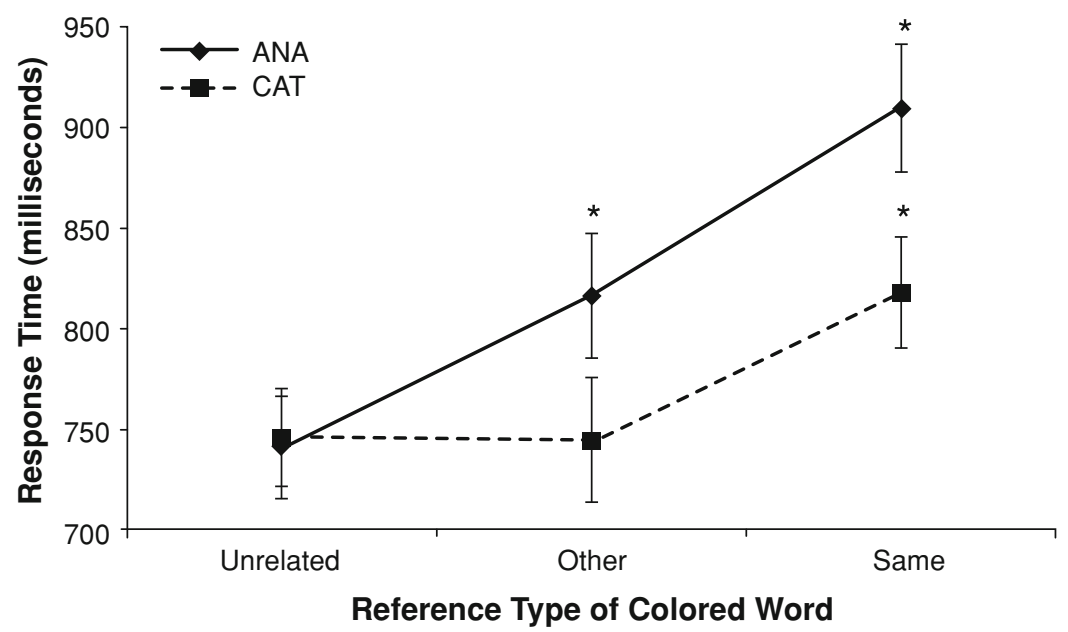

Figure 3. Mean response times (in milliseconds) for critical trials. Asterisks denote mean response times that were significantly slower than those for unrelated trials in the same instruction type, indicating that priming occurred. Error bars represent the standard error of the mean. 
instruction type $[t(17)=2.68, S E=20.39, p<.05]$ and that CAT-same trials $(M=818 \mathrm{msec})$ were significantly slower than unrelated trials $(M=746 \mathrm{msec})$ in the CAT instruction type $[t(17)=2.945, S E=20.29, p<.01]$, verifying that priming of categorical relations occurred in both ANA and CAT instruction types. RTs for ANAother trials were as slow as RTs for CAT-same trials $(t<$ 1 ), indicating that priming of categorical relations was as strong in the ANA instruction type as in the CAT instruction type.

ANA-same trials $(M=909 \mathrm{msec})$ showed significantly slower RTs than CAT-other trials $[M=744 \mathrm{msec} ; t(34)=$ 3.969, $S E=31.90, p<.001]$. RTs for CAT-other trials were not significantly slower than those for unrelated trials in the CAT instruction type $(t<1)$. Thus, priming of abstract analogical relations occurred in the ANA instruction type but not in the CAT instruction type even though these two trial types used identical stimuli. ANA-same trials also showed slower RTs than CAT-same trials $[t(34)=$ 2.10, $S E=43.59, p<.05]$.

Overall mean RTs for the ANA instruction type ( $M=$ $761 \mathrm{msec})$ and the CAT instruction type ( $M=748 \mathrm{msec})$ were not significantly different $(t<1)$. Also, unrelated trials did not differ between instruction types $(t<1)$. Therefore, differences between the ANA and CAT instruction types on critical trials cannot be attributed to inherent differences in speed of responding between the two groups of subjects.

\section{DISCUSSION}

Several important findings emerged to support the hypothesis that activation of category concepts is fundamental to reasoning analogically. Specifically, the one-to-one alignment of elements in analogical mapping seems to be categorical in nature (two elements are alignable when they are comembers of a common category). Our data indicate that activation of category concepts occurred automatically during analogical processing because categorical relations were primed in the ANA instruction type even though the subjects were not instructed to identify categorical relations. Indeed, our data indicate that categorical relations were activated as strongly during analogical reasoning (ANA instruction type) as they were when the subjects explicitly identified categorical relations (CAT instruction type).

Additionally, the findings of the present investigation provide empirical evidence to support the hypothesis that the representation of abstract analogical relations can be distinguished from specific terms that make up analogies. This is indicated by the significantly greater priming of analogical relations on ANA-same trials than on CATother trials despite the fact that the specific terms of the four-word sets (and the colored word that followed) were identical in the two instruction types. It is also indicated by the fact that priming of analogical relations was successfully measured using colored words that referred directly to the analogical relations themselves, rather than to the specific content terms (four words) of the analogy. Indeed, mental representations of abstract analogical relations seem to have demonstrated particularly strong activation, as evidenced by the fact that priming on ANA-same trials was stronger even than priming on CAT-same trials.

Two previous studies have suggested that abstract analogical relations are subject to priming. Spellman et al. (2001) found that subjects were faster at making lexical decisions about and naming a word pair when they had been presented with a previous word pair that represented a similar semantic relation. Spellman and colleagues also reported that analogical priming was not spontaneous, but only occurred when subjects were instructed to focus on the relations between the word pairs they received. This finding is consistent with the finding of the present investigation that abstract analogical relations were not primed when the subjects were not instructed to identify analogical relations between word pairs (CAT instruction type). Blanchette and Dunbar (2002) found that, after a delay, subjects falsely reported having read sentences in a text they were given if the content of those sentences had been primed by an analogous item that did appear in the text. The finding of analogical priming in both of these studies is consistent with the results of the present investigation. However, as noted by Spellman et al., these studies utilized "mediated" priming because the priming of the second item by the first item was assumed to proceed via the activation of an intermediate semantic link (an abstract analogical relation). The present investigation is the first to directly assess priming of the analogical relation itself.

The two main findings of the present investigation are supported by recent fMRI data. Bunge, Wendelken, Badre, and Wagner (2005) and Green et al. (2006) found that four-word analogies, but not nonanalogical four-word sets, elicit activation in the frontal pole of the brain. Outside of frontopolar cortex, however, the four-word analogy task and a four-word categorical task (the CAT instruction type) elicit virtually the same network of brain activations. Interpreted in the context of these previous findings, the strong priming of analogical relations in the ANA instruction type, but not the CAT instruction type, may be associated with activity in frontopolar cortex. The priming of categorical relations in the ANA and CAT instruction types may reflect the activation of a common cortical network involved in processing categorical relations for both analogical mapping and explicit categorization.

\section{Cognitive Processes of Analogy}

Figure 4 represents a decomposition of the hypothesized cognitive processes that the subjects performed during the four-word analogy task (ANA instruction type). The subjects first had to retrieve the individual meanings of each of the four words and then retrieve the conventionalized semantic relations within each word pair (what is the common relationship between these two words?). As discussed below, understanding the conventionalized semantic relation within a word pair requires understanding the individual roles that each word plays in this relation. Once they understood the relations within word pairs, the subjects had to properly align words between 


\section{Cognitive Components of Four-Word Analogy}

\begin{tabular}{|c|c|c|}
\hline \multirow{2}{*}{$\begin{array}{l}\text { Semantic retrieval } \\
\text { 1. Individual word } \\
\text { meanings } \\
\text { 2. Conventionalized } \\
\text { semantic relations } \\
\text { (within word pairs) }\end{array}$} & \begin{tabular}{|c} 
Categorical one-to-one \\
alignment of words \\
(between word pairs)
\end{tabular} & $\begin{array}{l}\text { Abstract relational } \\
\text { integration } \\
\text { (comprehension of } \\
\text { entire analogy) }\end{array}$ \\
\hline & $\begin{array}{c}\text { Priming of category } \\
\text { relations } \\
\text { (occurred as strongly in } \\
\text { ANA instruction type as in } \\
\text { CAT instruction type) }\end{array}$ & $\begin{array}{l}\text { Priming of abstract } \\
\text { analogical relations } \\
\text { (occurred in ANA } \\
\text { instruction type but not } \\
\text { CAT instruction type) }\end{array}$ \\
\hline
\end{tabular}

Figure 4. Componential sketch of the hypothesized cognitive processes involved in the analogy task (ANA instruction type).

the two word pairs subservient to analogical mapping. We hypothesized that this alignment proceeded on the basis of category comembership. This hypothesis was supported by the observed priming of category relations in the analogy instruction type. Finally, the analogical mapping process is completed when all the component relations are integrated into a single whole, such that the abstract analogical relation between the two word pairs is comprehended. The term abstract relational integration has been used to describe this component of analogical reasoning (Bunge et al., 2005; Green et al., 2006; Ramnani \& Owen, 2004). Consistent with our hypothesis, comprehension of the analogy led to priming of abstract analogical relations in the analogy instruction type.

\section{Theoretical Implications}

The four-word stimuli used in the present study were chosen because they allowed us to instantiate the ANA and CAT instruction types independently but with identical stimuli. One critical aspect of these stimuli was that terms could be categorized as tokens of a real-world type. For example, in the four-word set gun:bullet + bow:arrow, the terms gun and bow both fit into the category weapon, and bullet and arrow both fit into the category projectile. Alternatively, these terms can also be categorized by the roles they play within the context of the analogy (i.e., gun and bow are items that launch other items; bullet and arrow are items that are launched). On this categorical alignment strategy, two items are aligned if they are tokens of a functional type within the context of the analogy; that is, they are aligned not on the basis of what they are, but on the basis of what they do (what role they play) in the analogy (see Holyoak \& Thagard, 1997, for a discussion of role-based alignment).

The present stimuli did not allow us to clearly distinguish whether the subjects aligned terms on the basis of real-world type or on the basis of what role they played in the analogy. The difference between the category concepts that would be used in these two strategies is often subtle (e.g., weapons vs. items that launch projectiles). Thus, the colored words that followed four-word sets in
CAT-same trials generally would have been semantically related to the categorical relations formed through either strategy (e.g., the word weapon is semantically related both to the category weapons and the category items that launch projectiles). Accordingly, the observed priming of these colored words might have occurred under either categorization strategy. Nonetheless, whether the observed priming on ANA-other trials reflects categorization based on real-world type or based on role within the analogy, the fact that these category concepts were primed in the ANA instruction type indicates that some form of categorization occurred during analogical reasoning. Thus, the present research provides the first empirical finding of categorization as a mechanism for analogical mapping.

Because it was necessary to use categories based on real-world types, the analogies in the present investigation were all within-domain analogies. That is, the terms in the two word pairs were drawn from the same semantic domain. Further investigation will be necessary to assess priming of categorical relations in analogies whose structural elements cannot be categorized on the basis of the real-world types. Such analogies are referred to as cross-domain analogies (e.g., Bowdle \& Gentner, 2005) because the items in the respective word pairs are taken from disparate semantic domains. Categorization in crossdomain analogies would necessarily proceed on the basis of the roles that terms play within the analogy because categories based on real-world types would not apply to terms from disparate domains. Importantly, the information required to perform this categorization is available in cross-domain analogies (e.g., Gentner, 1983; Holyoak \& Thagard, 1995). In cross-domain analogies, as in other forms of analogies, it is impossible to understand the conventionalized semantic relations within each word pair without understanding the roles of the individual words within the word pair. For example, in the first half of the four-word set planet:sun + electron:nucleus, to understand that planet plays the role of "satellite" and sun plays the role of "orbited object," it is necessary to understand the conventionalized semantic relation "revolves around." Once the roles of the individual terms have been deter- 
mined within each word pair, it is possible to align terms between the two word pairs.

Alignment of terms that play similar roles has been suggested in previous models of analogical mapping (e.g., Bowdle \& Gentner, 2005; Gentner, 1983; Holyoak \& Thagard, 1995, 1997). However, these accounts address categorization only as an end result of determining that two items or situations are analogous. Bowdle and Gentner argued that forming a metaphor, taken as a form of analogical mapping, can lead to the development of category schemas. Glucksberg and Keysar (1990) argued that metaphors represent category relations between whole concepts (not component terms). The present research extends previous models of analogical mapping by demonstrating that, at least for within-domain analogies, categorization serves as a mechanism by which individual component terms are aligned subservient to analogical mapping. This finding leads to the hypothesis that analogical mapping in general operates through categorization.

\section{Conclusion}

The present study informs our understanding of how the mind structures and processes analogy. First, our findings suggest that the process of analogical mapping can be thought of as a process of categorization. In addition, the present findings empirically demonstrate that, when people comprehend the analogy between two items, strong activation of the abstract analogical relation itself occurs and can be directly assessed using the word that refers to the relation itself. These new insights into analogical reasoning provide for a more complete understanding of one of the most pervasive and quintessentially human forms of complex thought.

\section{REFERENCES}

Bassok, M., Chase, V. M., \& Martin, S. A. (1998). Adding apples and oranges: Alignment of semantic and formal knowledge. Cognitive Psychology, 35, 99-134.

BLANCHETTE, I., \& DUNBAR, K. (2002). Representational change and analogy: How analogical inferences alter target representations. Journal of Experimental Psychology: Learning, Memory, \& Cognition, 28, 672-685.

BowDLE, B. F., \& Gentner, D. (2005). The career of metaphor. Psychological Review, 112, 193-216.

Bunge, S. A., Wendelken, C., Badre, D., \& Wagner, A. D. (2005). Analogical reasoning and prefrontal cortex: Evidence for separable retrieval and integration mechanisms. Cerebral Cortex, 15, 239-249.

Catena, A., Fuentes, L. J., \& Tudela, P. (2002). Priming and interference effects can be dissociated in the Stroop task: New evidence in favor of the automaticity of word recognition. Psychonomic Bulletin \& Review, 9, 113-118.

Cohen, J. D., MacWhinney, B., Flatt, M., \& Provost, J. (1993). PsyScope: An interactive graphic system for designing and controlling experiments in the psychology laboratory using Macintosh computers. Behavior Research Methods, Instruments, \& Computers, 25, 257-271.
Dunbar, K., \& Blanchette, I. (2001). The in vivo/in vitro approach to cognition: The case of analogy. Trends in Cognitive Sciences, $\mathbf{5}$, 334-339.

GENTNER, D. (1983). Structure-mapping: A theoretical framework for analogy. Cognitive Science, 7, 155-170.

Gentner, D., \& MARKMAN, A. B. (1997). Structure mapping in analogy and similarity. American Psychologist, 52, 45-56.

Gentner, D., RattermanN, M. J., \& ForbUs, K. D. (1993). The roles of similarity in transfer: Separating retrievability from inferential soundness. Cognitive Psychology, 25, 524-575.

Gick, M. L., \& Holyoak, K. J. (1980). Analogical problem solving. Cognitive Psychology, 12, 306-355.

GiCK, M. L., \& HOLYOAK, K. J. (1983). Schema induction and analogical transfer. Cognitive Psychology, 15, 1-38.

GlucksberG, S., \& KeYSAR, B. (1990). Understanding metaphorical comparisons: Beyond similarity. Psychological Review, 97, 3-18.

Green, A., Fugelsang, J., Kraemer, D. J. M., Shamosh, N., \& DunBAR, K. (2006). The dynamic role of prefrontal cortex in complex reasoning and abstract thought. Manuscript submitted for publication.

Hesse, M. (1966). Models and analogies in science. Notre Dame, IN: University of Notre Dame Press.

Holyoak, K. J. (2005). Analogy. In K. J. Holyoak \& R. G. Morrison (Eds.), Cambridge handbook of thinking and reasoning (pp. 117142). Cambridge: Cambridge University Press.

HoLYOAK, K. J., \& KoH, K. (1987). Surface and structural similarity in analogical transfer. Memory \& Cognition, 15, 332-340.

Holyoak, K. J., \& Thagard, P. (1995). Mental leaps: Analogy in creative thought. Cambridge, MA: MIT Press.

Holyoak, K. J., \& Thagard, P. (1997). The analogical mind. American Psychologist, 52, 35-44.

KeANE, M. (1988). Analogical problem solving. Chichester, U.K.: Ellis Horwood.

Marí-Beffa, P., Estévez, A. F., \& Danziger, S. (2000). Stroop interference and negative priming: Problems with inferences from null results. Psychonomic Bulletin \& Review, 7, 499-503.

Markman, A. B., \& Gentner, D. (2000). Structure-mapping in the comparison process. American Journal of Psychology, 113, 501-538.

Pritchard, V. E., \& NEUMANN, E. (2004). Negative priming effects in children engaged in nonspatial tasks: Evidence for early development of intact inhibitory mechanisms. Developmental Psychology, 40, 191-203.

RAMNANI, N., \& OWEN, A. M. (2004). Anterior prefrontal cortex: Insights into function from anatomy and neuroimaging. Nature Reviews Neuroscience, 5, 184-194.

Ross, B. H. (1989). Distinguishing types of superficial similarities: Different effects on the access and use of earlier problems. Journal of Experimental Psychology: Learning, Memory, \& Cognition, 15, 456-468.

SchunN, C. D., \& Dunbar, K. (1996). Priming, analogy, and awareness in complex reasoning. Memory \& Cognition, 24, 271-284.

Segal, Z. V., Gemar, M., Truchon, C., Guirguis, M., \& Horowitz, L. M. (1995). A priming methodology for studying self-representation in major depressive disorder. Journal of Abnormal Psychology, 104, 205-213.

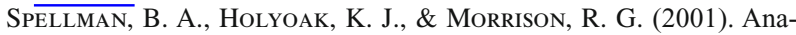
logical priming via semantic relations. Memory \& Cognition, 29, 383-393.

STERNBERG, R. (1977). Intelligence, information processing, and analogical reasoning. Hillsdale, NJ: Erlbaum.

Wharton, C. M., Holyoak, K. J., \& Lange, T. E. (1996). Remote analogical reminding. Memory \& Cognition, 24, 629-643.

WISNIEWSKI, E. J., \& BASSOK, M. (1999). What makes a man similar to a tie? Stimulus compatibility with comparison and integration. Cognitive Psychology, 39, 208-238. 
APPENDIX

Stimuli for Critical Trials

\begin{tabular}{|c|c|c|}
\hline Four-Word Set & $\begin{array}{l}\text { Colored Word Referring } \\
\text { to Categorical Relation }\end{array}$ & $\begin{array}{l}\text { Colored Word Referring } \\
\text { to Analogical Relation }\end{array}$ \\
\hline Soda:Can+Beer:Bottle & Beverage & Contain \\
\hline Driver:Taxi+Chauffeur:Limousine & Automobile & Drive \\
\hline Mechanic:Wrench+Carpenter:Hammer & Worker & Use \\
\hline Novel:Novelist+Poem:Poet & Literature & Write \\
\hline Cub:Bear+Kitten:Cat & Baby & Become \\
\hline Gun:Bullet+Bow:Arrow & Weapon & Shoot \\
\hline Marinara:Spaghetti + Alfredo:Fettuccini & Sauce & Cover \\
\hline Vine:Grape+Tree:Orange & Fruit & Grow \\
\hline Oven:Casserole +Stove:Soup & Food & Cook \\
\hline Song:Stereo+Movie:VCR & Device & Play \\
\hline Waiter:Customer+Teller:Client & Employee & Serve \\
\hline Axe:Trunk+Saw:Branch & Tool & Cut \\
\hline Pitcher:Baseball+Quarterback:Football & Athlete & Throw \\
\hline Fish:Caviar+Bird:Egg & Animal & Lay \\
\hline Painter:Portrait+Sculptor:Sculpture & Artist & Create \\
\hline Tuxedo:Groom + Gown:Bride & Clothing & Wear \\
\hline
\end{tabular}

(Manuscript received December 22, 2004;

revision accepted for publication June 30, 2005.) 